ИЗВЕСТИЯ АКАДЕМИИ НАУК ЭСТОНСКОП ССР. ФИЗИКА * МАТЕМАТИКА PROCEEDINGS OF THE ACADEMY OF SCIENCES OF THE ESTONIAN SSR. PHYSICS * MATHEMATICS

$1985,34,2$

\title{
Юлле КОТTA
}

\section{ИССЛЕДОВАНИЕ ВЗАИМОСВЯЗЕЙ МНОГОМЕРНОЙ БИЛИНЕИНОЙ СИСТЕМЫ}

\author{
(Представил Н. Алумяэ)
}

1. Введение. При проектировании систем автоматического управления многомерными объектами возникает вопрос о существовании автономных вход-выход пар. Если линейный многомерный объект описан матричной передаточной функцией, то наличие связей между различными вход-выход парами определяется существованием ненулевых элементов вне главной диагонали в этой матрице. Для линейных систем, описываемых уравнениями состояния, попытка решить эту задачу сделана в $\left[{ }^{1}\right]$, где получено необходимое (но не достаточное) условие автономности некоторой вход-выход пары от остальных входов и выходов системы. Основным понятием разработанного в [ $\left.{ }^{1}\right]$ метода является относительная переходная функция разомкнутой и частично замкнутой подсистем. Относительная переходная функция рассматриваемой вход-выход пары позволяет судить о взаимосвязанности этой пары с остальными вход-выход переменными многомерного объекта.

В настоящей работе метод из $\left.{ }^{1}\right]$ обобщен для билинейных систем специального класса - для т. н. диадических билинейных систем. В отличне от определения линейных систем для определения билинейной системы не достаточно задавать ее переходную функцию. Показывается, что для определения диадической билинейной системы надо кроме переходной функции задавать еще переходную функцию линейной части этой системы. Из этого следует, что для изучения взаимосвязанности диадической билинейной системы целесообразно анализировать поведение двух относительных переходных функций.

Рассматриваемый подкласс билинейных систем привлекает много внимания. Большинство работ посвящается анализу управляемости $\left[{ }^{2-7}\right]$. Менее изучены вопросы оптимизации $\left[{ }^{8}\right]$, стабилизации $\left[{ }^{9}\right]$ и обратимости $[10,11]$.

2. Диадические билинейные системы. Пусть уравнения билинейной системы заданы в виде

$$
\begin{aligned}
& x(t+1)=A x(t)+\sum_{j=1}^{m} B_{j} x(t) u_{j}(t)+G u(t), \\
& y(t)=C x(t),
\end{aligned}
$$

где $x \in R^{n}, u=\left[u_{1} \ldots u_{m}\right] \in R^{m}, y=\left[y_{1} \ldots y_{m}\right] \in R^{m}$, а $A, G, C, B_{j}$, $j=1, \ldots, m-$ постоянные матрицы. Билинейные системы (1), которые удовлетворяют условию

$$
\text { ранг }\left[B_{j} g_{j}\right]=1, \quad j=1, \ldots, m,
$$


где $g_{j}$ есть $j$-й столбец матрицы $G$, называются диадическими билинейными. Из (2) следует, что $B_{j}$ можно разложить следующим образом:

$$
B_{j}=g_{j} d_{j}^{T},-
$$

где $d_{j}-n$-мерный вектор. Следовательно, уравнения системы (1) можно переписать

$$
\begin{aligned}
& x(t+1)=A x(t)+G \Gamma(x) u(t), \\
& y(t)=C x(t),
\end{aligned}
$$

где $\Gamma(x)=\operatorname{diag}\left\{\gamma_{1}(x) \ldots \gamma_{m}(x)\right\}, \gamma_{j}(x)=d_{j}^{T} x(t)+1, j=1, \ldots, m$.

3. Определение диадической билинейной системы по двум переходным функциям. Билинейная система полностью описывается совокупностью ядер Вольтерра. В настоящем разделе показывается, что ядра Вольтерра диадической билинейной системы с одним входом и одним выходом можно определить по двум переходным функциям, а именно, по переходным функциям билинейной системы и линейной части этой системы. Это свойство диадических билинейных систем в дальнейшем используется при анализе взаимосвязанности вход-выход пар многомерного билинейного объекта.

Пусть даны переходная функция

$$
p(t)=[y(t) \mid x(0)=0, u(t)=1, t=0,1, \ldots]
$$

диадической билинейной системы с одним входом и одним выходом

$$
\begin{aligned}
& x(t+1)=A x(t)+g d^{T} x(t) u(t)+g u(t) ; \\
& y(t)=c^{T} x(t)
\end{aligned}
$$

и переходная функция

$$
q(t)=[y(t) \mid x(0)=0, u(t)=1, t=0,1, \ldots]
$$

линейной части той же системы

$$
\begin{aligned}
& x(t+1)=A x(t)+g u(t), \\
& y(t)=c^{T} x(t) .
\end{aligned}
$$

Покажем, что функции $p(t)$ и $q(t)$ определяют все ядра Вольтерра системы (4). $n$-е $(n=1,2, \ldots)$ регулярное ядро Вольтерра системы (4) выражается через матрицы $A, c, d$ и $g$ следующим образом $\left[{ }^{12}\right]$ :

$$
h_{n}\left(t_{1}, \ldots, t_{n}\right)=c^{T} A^{t_{n}-1} g d^{T} A^{t_{n-1}-1} g \ldots d^{T} A^{t_{1}-1} g .
$$

Введем обозначения

$$
\begin{gathered}
v(t)=c^{T} A^{t-1} g, \\
w(t)=d^{T} A^{t-1} g .
\end{gathered}
$$

Отсюда видно, что ядра Вольтерра определены, если известны функции $v(t)$ и $w(t)$. Далее будет показано, как эти функции могут быть рекуррентно выражены через переходные функции $p(t)$ и $q(t)$.

Из уравнений (5) получим

$$
q(t)=\sum_{k=1}^{t} c^{T} A^{k-1} g=\sum_{k=1}^{t} v(k) .
$$

Следовательно, 
Далее, нз уравнений (4) получим

$$
v(t)=q(t)-q(t-1) .
$$

$$
\begin{aligned}
p(t) & =\sum_{k=1}^{t} c^{T}\left(A+g d^{T}\right)^{k-1} g= \\
& =\sum_{k=1}^{t} \sum_{t_{1}+\ldots+t_{k}=k}^{t} v\left(t_{k}\right) w\left(t_{k-1}\right) \ldots w\left(t_{1}\right)= \\
& =p(t-1)+v(1) w^{t-1}(1)+\sum_{k=1}^{t-1} \sum_{t_{1}+\ldots+t_{k}=t} v\left(t_{k}\right) w\left(t_{k-1}\right) \ldots w\left(t_{1}\right) .
\end{aligned}
$$

Выражаем

$$
w(t-1)=\left\{\begin{array}{l}
\frac{p(t)-p(t-1)-v(t)}{v(1)},-t=2 \\
\frac{1}{v(1)}[p(t)-p(t-1)-v(t)-v(1) w(1)- \\
\left.-\sum_{\substack{t_{1}+t_{2}=t \\
t_{1} \neq t-1 \\
t=3,4}} v\left(t_{k}\right) w\left(t_{1}\right)-\sum_{k=3}^{t-1} \sum_{t_{1}+\ldots+t_{k}=t} v\left(t_{k}\right) w\left(t_{k-1}\right) \ldots w\left(t_{1}\right)\right],
\end{array}\right.
$$

Отсюда видно, что $(t), t=1,2, \ldots$ рекуррентно вычисляемы по $p(t)$ и $v(t)$.

4. Относительные переходные функции. Для исследования взаимосвязанности пары $\left(y_{i}, u_{j}\right)$ с остальными входами-выходами определим функции взаимосвязанности $\Phi_{i j}(t)$ и $\psi_{i j}(t)$ как относительные переходные функции системы (3) и ее линейной части соответственно. Определение и формула вычисления относительной переходной функции $\psi_{i j}(t)$ на основе уравнений состояния системы даны в [ $\left.{ }^{1}\right]$

$$
\psi_{i j}(t)=\frac{\sum_{k=1}^{t} c_{i}^{T} A^{k-1} g_{j}}{\sum_{k=1}^{t} c_{i}^{T}\left(M_{i j} A\right)^{k-1} M_{i j} g_{j}},
$$

где $M_{i j}=I-G_{j}\left(C_{i} G_{j}\right)^{-1} C_{i}, \quad$ a $G_{j}$ - матрица $G$ без $j$-го столбца, $C_{i}-$ матрица $C$ без $i$-й строки, $c_{i}{ }^{T}-i$-я строка матрицы $C$.

Дадим определение относительной переходной функции $\Phi_{i j}(t)$. Для выделения пары $\left(y_{i}, u_{j}\right)$ перепишем (3) в виде

$$
\begin{gathered}
x(t+1)=A x(t)+G_{j} \Gamma_{j}(x) \bar{u}_{j}(t)+g_{j} \gamma_{j}(x) u_{j}(t), \\
\bar{y}_{i}(t)=C_{i} x(t), \\
y_{i}(t)=c_{i}^{T} x(t) ;
\end{gathered}
$$

где $\bar{u}_{j}-(m-1)$-мерный вектор управляющих воздействий без. $u_{j}$, $\bar{y}_{i}-(m-1)$-мерный вектор выходов без $y_{i}, \Gamma_{j}(x)$ - матрица $\Gamma(x)$ без $j$-й строки и $j$-го столбца. Относительная переходная функция $\Phi_{i j}(t)$ пары $\left(y_{i}, u_{j}\right)$ определяется как отношение двух переходных функций

$$
\Phi_{i j}(t)=p_{i j}(t) / q_{i j}(t), \quad t=1,2, \ldots,
$$

где 


$$
p_{i j}(t)=\left[y_{i}(t) \mid x(0)=0, u_{j}(t)=1, \bar{u}_{j}(t)=0\right]
$$

переходная функция пары $\left(y_{i}, u_{j}\right)$ при $\bar{u}_{j}(t)=0$ (переходная функция разомкнутой подсистемы),

$$
q_{i j}(t)=\left[y_{i}(t) \mid x(0)=0, u_{j}(t)=1, \bar{y}_{i}(t)=0\right]
$$

переходная функция пары $\left(y_{i}, u_{j}\right)$ при $\bar{y}_{j}(t)=0$ (переходная функция частично замкнутой подсистемы). Задача состоит теперь в определении функций $p_{i j}(t)$ и $q_{i j}(t)$ на основе уравнений состояния.

Из уравнения (7) получим

$$
p_{i j}(t)=\sum_{k=1}^{t} c_{i}^{T}\left(A+B_{j}\right)^{k-1} g_{j}
$$

Для определения переходной функции $q_{i j}(t)$ частично замкнутой системы требуются уравнения «идеального» регулятора, обеспечивающего выполнение $\bar{y}_{i}(t)=0$ при $u_{j}(t)=1, t=1,2, \ldots$. В разделе 6 будет показано, что таким «идеальным» регулятором является обратная система (7), (8), где $u_{j}$ считается не управляющим воздействием, а возмущением.

5. Система, обратная к билинейной системе с возмущением. Обратной называется система, которая по выходу данной системы восстанавливает значение входа. Выводим уравнения системы, обратной к билинейной системе с возмущением

$$
\begin{aligned}
& x(t+1)=A x(t)+G \Gamma(x) u(t)+d(t), \\
& y(t)=C x(t) .
\end{aligned}
$$

Уравнение (10) можно переписать в виде

$$
\left[\begin{array}{cc}
I & -G \Gamma(x) \\
0 & C G \Gamma(x)
\end{array}\right]\left[\begin{array}{c}
x(t+1) \\
u(t)
\end{array}\right]=\left[\begin{array}{c}
A x(t)+d(t) \\
y(t+1)-C A x(t)-C d(t)
\end{array}\right] .
$$

Желаемая обратная система - это решение (11) относительно пары векторов $[x(t+1), u(t)]$. Известно, что решение существует и оно единственно тогда и только тогда, когда матрица

$$
R=\left[\begin{array}{ll}
I & -G \Gamma(x) \\
0 & C G \Gamma(x)
\end{array}\right]
$$

неособенная. Если матрица $R$ неособенная, то с учетом формулы өбращения блочной матрицы получим

$$
R^{-1}=\left[\begin{array}{cc}
I & G(C G)^{-1} \\
0 & \Gamma^{-1}(x)(C G)^{-1}
\end{array}\right] .
$$

С учетом (11) и (12) получим уравнения обратной динамической системы в виде

$$
\begin{aligned}
& x(t+1)=\tilde{A} x(t)+\widetilde{B} y(t+1)+M d(t), \\
& u(t)=C(x) x(t)+D(x) y(t+1)+E(x) d(t),
\end{aligned}
$$

где

$$
\begin{array}{ll}
M=I-G(C G)^{-1} C, & C(x)=-\Gamma^{-1}(x)(C G)^{-1} C A, \\
A=M A, & D(x)=\Gamma^{-1}(x)(C G)^{-1}, \\
\widetilde{B}=G(C G)^{-1}, & E(x)=-\Gamma^{-1}(x)(C G)^{-1} C .
\end{array}
$$


6. Уравнение «идеального» регулятора. На основё результатов, изложенных в разделе 5 , обратная для системы (7), (8) имеет вид

$$
\begin{aligned}
x(t+1) & =M_{i j}\left[A+g_{j} d_{j}^{T} u_{j}(t)\right] x(t)+G_{j}\left(C_{i} G_{j}\right)^{-1} \bar{y}_{i}(t+1)+ \\
& +M_{i j} g_{j} u_{j}(t), \\
\bar{u}_{j}(t) & =-\Gamma_{j}^{-1}(x)\left(C_{i} G_{j}\right)^{-1} C_{i}\left[A+g_{j} d_{j}^{T} u_{j}(t)\right] x(t)+ \\
& +\Gamma_{j}^{-1}(x)\left(C_{i} G_{j}\right)^{-1} \bar{y}_{i}(t+1)- \\
& -\Gamma_{j}^{-1}(x)\left(C_{i} G_{j}\right)^{-1} C_{i} g_{j} u_{j}(t) .
\end{aligned}
$$

В данном разделе покажем, что обратная система (14) с отрицательной обратной связью обеспечивает $\bar{y}_{i}(t)=0$ при любых $u_{j}(t), t=1,2, \ldots$. Для этого выпишем уравнения частично замкнутой системы. Подставляя $\bar{u}_{j}(t)$ из $(14)$ в $(7)$, получим

$$
\left[I+G_{j}\left(C_{i} G_{j}\right)^{-1} C_{i}\right] x(t+1)=M_{i j} A x(t)+M_{i j} g_{j \gamma_{j}}(x) u_{j}(t) .
$$

Если матрица $I+G_{j}\left(C_{i} C_{j}\right)^{-1} C_{i}$ неособенная, то существует обратная матрица $1-1 / 2 G_{j}\left(C_{i} C_{j}\right)^{-1} C_{i}$ и (15) можно переписать в виде

$$
x(t+1)=\left[I-1 / 2 G_{j}\left(C_{i} G_{j}\right)^{-1} C_{i}\right]\left[M_{i j} A x(t)+M_{i j} g_{j \gamma_{j}}(x) u_{j}(t)\right] .
$$

Поскольку

$$
\left[I-1 / 2 G_{j}\left(C_{i} G_{j}\right)^{-1} C_{i}\right] M_{i j}=M_{i j}
$$

то получим уравнение состояния частично замкнутой системы в виде

$$
x\left(t_{+}+1\right)=M_{i j} A x(t)+M_{i j} g_{j} \gamma_{j}(x) u_{j}(t) .
$$

Исследуем выход $\bar{y}_{i}(t)$. Так как $\bar{y}_{i}(t)=C_{i} x(t)$ и в силу того, что $C_{i} M_{i j}=0$, то из (16) получим $\bar{y}_{i}(t)=0, t=1,2 \ldots$. Следовательно, система (14) является «идеальным» регулятором в определенном выше смысле.

7. Вычисление коэффициента взаимосвязанности $\Phi_{i j}(t)$. Поскольку $y_{i}(t)=c_{i}{ }^{T} x(t)$, из (16) при нулевых начальных условиях $x(0)=0$ получим

$$
q_{i j}(t)=\sum_{k=1}^{t} c_{i}^{T}\left[M_{i j}\left(A+B_{j}\right)\right]^{k-1} M_{i j} g_{j} .
$$

Следовательно, на основе параметров билинейного объекта (7)-(9) можно для любой пары $\left(y_{i}, u_{j}\right), i, j=1, \ldots, m$ (если только соответствующие обратные системы существуют) вычислить относительную переходную функцию

$$
\Phi_{i j}(t)=\frac{\sum_{k=1}^{t} c_{i}^{T}\left(A+B_{j}\right)^{k-1} g_{j}}{\sum_{k=1}^{t} c_{i}^{T}\left[M_{i j}\left(A+B_{j}\right)\right]^{k-1} M_{i j} g_{j}} .
$$

8. Условие автономности вход-выход пары. Докажем интуитивно очевидное утверждение, что для автономной вход-выход пары выполняется $\Phi_{i j}(t)=1$. Пусть скалярная система

$$
\begin{aligned}
& x_{1}(t+1)=A_{1} x_{1}(t)+B_{1} x_{1}(t) u_{1}(t)+g_{1} u_{1}(t), \\
& y_{1}(t)=c_{1}^{T} x_{1}(t)
\end{aligned}
$$


и система с $m-1$ входами й выходами

$$
\begin{aligned}
& x(t+1)=A x(t)+\sum_{j=2}^{m} B_{j} x(t) u_{j}(t)+G u(t), \\
& y(t)=C x(t)
\end{aligned}
$$

являются независимыми, автономными. Тогда уравнения системы, составленные из уравнений независимых подсистем (18) и (19), получим в виде

$$
\begin{gathered}
{\left[\begin{array}{cc}
x_{1} & (t+1) \\
x & (t+1)
\end{array}\right]=\left[\begin{array}{cc}
A_{1} & 0 \\
0 & A
\end{array}\right]\left[\begin{array}{c}
x_{1}(t) \\
x(t)
\end{array}\right]+\left[\begin{array}{cc}
B_{1} & 0 \\
0 & 0
\end{array}\right]\left[\begin{array}{c}
x_{1}(t) \\
x(t)
\end{array}\right] u_{1}(t)+} \\
+\sum_{j=2}^{m}\left[\begin{array}{ll}
0 & 0 \\
0 & B_{j}
\end{array}\right]\left[\begin{array}{c}
x_{1}(t) \\
x(t)
\end{array}\right] u_{j}(t)+\left[\begin{array}{cc}
g_{1} & 0 \\
0 & G
\end{array}\right]\left[\begin{array}{c}
u_{1}(t) \\
u(t)
\end{array}\right] \\
{\left[\begin{array}{l}
y_{1}(t) \\
y(t)
\end{array}\right]=\left[\begin{array}{cc}
c_{1}^{T} & 0 \\
0 & C
\end{array}\right]\left[\begin{array}{c}
x_{1}(t) \\
x(t)
\end{array}\right]}
\end{gathered}
$$

Очевидно,

$$
p_{11}(t)=\sum_{k=1}^{t}\left[\begin{array}{ll}
c_{1}^{T} & 0
\end{array}\right]\left[\begin{array}{cc}
A_{1}+B_{1} & 0 \\
0 & A
\end{array}\right]^{k-1}\left[\begin{array}{c}
g_{1} \\
0
\end{array}\right]=\sum_{k=1}^{t} c_{1}^{T}\left(A_{1}+B_{1}\right)^{k-1} g_{1} .
$$

Вычислим

$$
M_{11}=I-\left[\begin{array}{l}
0 \\
G
\end{array}\right]\left\{\left[\begin{array}{ll}
0 & C
\end{array}\right]\left[\begin{array}{l}
0 \\
G
\end{array}\right]\right\}^{-1}\left[\begin{array}{ll}
0 & C
\end{array}\right]=\left[\begin{array}{cc}
I & 0 \\
0 & I-G(C G)^{-1} C
\end{array}\right]
$$

и переходную функцию

$$
\begin{aligned}
q_{11}(t) & =\sum_{k=1}^{t}\left[\begin{array}{ll}
c_{1}^{T} & 0
\end{array}\right]\left[\begin{array}{cc}
\left(A_{1}+B_{1}\right)^{k-1} & 0 \\
0 & {\left[\left[I-G(C G)^{-1} C\right] A\right]^{k-1}}
\end{array}\right]\left[\begin{array}{c}
g_{1} \\
0
\end{array}\right]= \\
& =\sum_{k=1}^{t} c_{1}^{T}\left(A_{1}+B_{1}\right)^{k-1} g_{1} .
\end{aligned}
$$

Из (20) и (21) получим $p_{11}(t)=q_{11}(t)$, и следовательно, относительная переходная функция $\Phi_{11}(t)=1$.

Из $\left[{ }^{1}\right]$ известно, что если пара $\left(y_{i}, u_{j}\right)$ является автономной, то коэффициент $\psi_{i j}(t)$ тоже равен единице. Если значения функций $\Phi_{i j}(t)$ и $\psi_{i j}(t)$ значительно отличаются от единицы, то рассматриваемая пара имеет сильную взаимосвязь с другими входами-выходами.

Следует отметить, что $\Phi_{i j}(t)=1, \psi_{i j}(t)=1$ является только необходимым условием автономности.

9. Пример. Применим разработанную методику для определения автономных пар входов и выходов многомерного диадического билинейного объекта. Рассмотрим систему

$$
\begin{gathered}
x(t+1)=A x(t)+B_{1} x(t) u_{1}(t)+B_{2} x(t) u_{2}(t)+g_{1} u_{1}(t)+g_{2} l_{2}(t), \\
y_{1}(t)=c_{1}^{T} x(t), \\
y_{2}(t)=c_{2}^{T} x(t), \\
A=\left[\begin{array}{rr}
0,504 & 0,021 \\
-0,021 & 0,396
\end{array}\right], \quad B_{1}=g_{1} h_{1}^{T}=\left[\begin{array}{r}
1,042 \\
-0,208
\end{array}\right]\left[\begin{array}{ll}
0,3 & 0,06
\end{array}\right]=
\end{gathered}
$$




$$
\begin{aligned}
& =\left[\begin{array}{rr}
0,313 & 0,063 \\
-0,063 & -0,013
\end{array}\right], \quad B_{2}=g_{2} h_{2}^{T}=\left[\begin{array}{r}
-0,417 \\
2,083
\end{array}\right]\left[\begin{array}{ll}
0,1 & 0,5
\end{array}\right]= \\
& =\left[\begin{array}{rr}
-0,042 & -0,208 \\
0,208 & 1,042
\end{array}\right], . \\
& c_{1}^{T}=\left[\begin{array}{ll}
1 & 0,2
\end{array}\right], \quad c_{2}^{T}=\left[\begin{array}{ll}
0,2 & 1
\end{array}\right] .
\end{aligned}
$$

Относительные переходные функции этой системы $\Phi_{11}(t), \Phi_{22}(t)$, $\psi_{11}(t), \psi_{22}(t)$ имеют значение 1. Таким образом, можно ожидать, что пары входов и выходов $\left(y_{1}, u_{1}\right)$ и $\left(y_{2}, u_{2}\right)$ являются автономными. Действительно, с помощью преобразования базиса в пространстве состояния

$$
\tilde{x}=T x,
$$

где $T=\left[\begin{array}{cc}1 & 0,2 \\ 0,2 & 1\end{array}\right],-$

уравнения системы приводятся к виду

$$
\begin{aligned}
& \tilde{x}(t+1)=\widetilde{x} \widetilde{x}(t)+\widetilde{B}_{1} \tilde{x}(t) u_{1}(t)+\widetilde{B}_{2} \tilde{x}(t) u_{2}(t)+\widetilde{g}_{1} u_{1}(t)+\widetilde{g}_{2} u_{2}(t), \\
& y_{1}=\tilde{c}_{1}^{T} \tilde{x}(t), \\
& y_{2}=\tilde{c}_{2}^{T} \tilde{x}(t),
\end{aligned}
$$

где

$$
\begin{gathered}
\widetilde{A}=\left[\begin{array}{cc}
0,5 & 0 \\
0 & 0,4
\end{array}\right], \quad \widetilde{B}_{1}=\widetilde{g}_{1} h_{1}^{T}=\left[\begin{array}{l}
1 \\
0
\end{array}\right]\left[\begin{array}{ll}
0,3 & 0
\end{array}\right]=\left[\begin{array}{cc}
0,3 & 0 \\
0 & 0
\end{array}\right], \\
\widetilde{B}_{2}=\widetilde{g}_{2} h_{2}^{T}=\left[\begin{array}{l}
0 \\
2
\end{array}\right]\left[\begin{array}{ll}
0 & 0,5
\end{array}\right]=\left[\begin{array}{ll}
0 & 0 \\
0 & 1
\end{array}\right], \\
\tilde{c}_{1}^{T}=\left[\begin{array}{ll}
1 & 0
\end{array}\right], \quad \tilde{c}_{2}^{T}=\left[\begin{array}{ll}
0,1
\end{array}\right] .
\end{gathered}
$$

Из этих уравнений видно, что пары $\left(y_{1}, u_{1}\right)$ и $\left(y_{2}, u_{2}\right)$ действительь автономны и систему можно разложить на две скалярные подсистемы.

\section{ЛИТЕ РАТ У РА}

1. Яаксоо Ю. И. В кн.: Исследования по теории многосвязных систем. М., «Наука», $1982,61-65$.

2. Mullis, C. T. IEEE Trans. Automat. Contr., 18, № 6, 608-615 (1973).

3. Goka, T., Tarn, T. J., Zaborszky, J. Automatica, 9, № 5, 615-622 (1973).

4. Evans, M. E., Murthy, D. N. P. IEEE Trans. Automat. Contr., 22, № 1, 78-83 (1977)

5. Evans, M. E., Murthy, D. N. P. Automatica, 14, № 2, 147-151 (1978).

6. Funahashi, Y. IEEE Trans. Automat. Contr., 24, № 4, 667-668 (1979).

7. Hollis, P., Murthy, D. N. P. Int. J. Syst. Sci, 12, № 4, 485-494 (1981).

8. Swamy, K. N., Tarn, T. J. Automatica, 15, № 6, 677-682 (1979).

9. Gutman, P.-O. IEEE Trans. Automat. Contr., 26, № 4, 917-922 (1981).

10. Hsu, C. S., Mohler, R. R. Trans ASME. J. Dyn. Systems, Measurem., and Contr., 102, № 2, 103-105 (1981).

11. Kotta, O. ENSV TA Toim. Füüs. Matem., 32, № 3, 323-326 (1983).

12. Mitzel, G. E., Clancy, S. J., Rugh, W. J. IEEE Trans. Automat. Contr., 24, № 2, 242-249 (1979).

Ннститут кибернетики

Академии наук Эстонской ССР
Поступила в редакцию 10/XI 1983 


\section{MITMEMÕTTMELISE BILINEAARSE SUSTEEMI SEOTUSE ANALUUS}

Töös ['] esitatud meetod lineaarse süsteemi seotuse analüüsiks on üldistatud diaadiliste bilineaarsete süsteemide klassile. Nimetatud meetod baseerub suhtelise siirdefunktsiooni môistel. Erinevalt lineaarsetest süsteemidest ei ole bilineaarne süsteem oma siirdefunktsiooniga määratud. On näidatud, et diaadiliste bilineaarsete süsteemide määramiseks on süsteemi siirdefunktsioonile lisaks vaja teada veel süsteemi lineaarse osa siirdefunktsiooni ja et seotuse analüüsiks tuleb vaadelda kahe suhtelise siirdefunktsiooni käitumist.

\section{Ulle KOTTA}

\section{INTERACTION ANALYSIS OF MULTIVARIABLE BILINEAR SYSTEM}

In this paper the method proposed in [ $\left.{ }^{1}\right]$ for interaction analysis of multivariable linear system is generalized to bilinear systems described by equations

$$
\begin{aligned}
& x(t+1)=A x(t)+\sum_{j=1}^{m}\left[B_{j} x(t)+g_{j}\right] u_{j}(t), \\
& y_{i}(t)=c_{i}^{T} x(t), \quad i=1, \ldots, m, \\
& \operatorname{rank}\left[B_{j} g_{j}\right]=1, \quad j=1, \ldots, m .
\end{aligned}
$$

The method for the linear system is based on the concept of a relative step response function. The relative step response function of the pair $\left(y_{i}, u_{j}\right)$ of the bilinear system is defined as the ratio of two step response functions

$$
\Phi_{i j}(t)=p_{i j}(t) / q_{i j}(t), \quad t=1,2, \ldots,
$$

where

$$
p_{i j}(t)=\left[y_{i}(t) \mid x(0)=0, u_{j}(t)=1, u_{k}(t)=0, k \neq j\right]
$$

is the step response function of the pair $\left(y_{i}, u_{j}\right)$ in the open loop system and

$$
q_{i j}(t)=\left[y_{i}(t) \mid x(0)=0, u_{j}(t)=1, y_{h}(t)=0, k \neq i\right]
$$

is the step response function of the pair $\left(y_{i}, u_{j}\right)$ in the closed loop system. Bilinear systems are not determined by their step response functions. It is shown in the paper that for determining the dyadic bilinear system, the step response function of the linear part of the system is needed in addition to the step response function of the system. Consequently, two relative response functions $\Phi_{i j}(t)$ and $\Psi_{i j}(t)$ (relative step response function of the linear part of the sysţem) must be studied for the interaction analysis of the dyadic bilinear system. It is shown that for an independent pair $\left(y_{i}, u_{j}\right)$, $\Phi_{i j}(t)=\Psi_{i j}(t)=1, t=1,2, \ldots$. 